Alana Barbosa Rodrigues ${ }^{\mathrm{a}}$

(iD) https://orcid.org/0000-0002-7681-1390

Vilma Sousa Santana ${ }^{b}$

(iD) https://orcid.org/0000-0003-3399-7612

a Secretaria de Estado da Saúde do

Tocantins. Palmas, TO, Brasil.

b Universidade Federal da Bahia, Instituto de Saúde Coletiva. Salvador, BA, Brasil.

Contato:

Alana Barbosa Rodrigues

E-mail:

alanarodrigues88@gmail.com

Artigo baseado na dissertação de mestrado intitulada Acidentes de trabalho fatais no estado do Tocantins, Brasil: oportunidades perdidas de prevenção, da autora Alana Barbosa Rodrigues, defendida no Instituto de Saúde Coletiva da Universidade Federal da Bahia (UFBA), Salvador, 2017.

Este trabalho não foi apresentado em reunião científica.

As autoras declaram não have conflitos de interesses e que o trabalho não foi subvencionado.

\section{Acidentes de trabalho fatais em Palmas, Tocantins, Brasil: oportunidades perdidas de informação}

\author{
Fatal work accidents in Palmas, Tocantins, Brazil: missed \\ opportunities of information
}

\section{Resumo}

Objetivo: estimar o sub-registro de acidentes de trabalho fatais (ATF) nos sistemas de informação do Ministério da Saúde, Secretaria Estadual de Segurança Pública (SSP), Ministério do Trabalho e Secretaria da Previdência Social, de 2007 a 2015, em Palmas, Tocantins. Métodos: para identificação dos casos de ATF, as fontes de dados foram o Sistema de Informação sobre Mortalidade (SIM), o Sistema de Informações Hospitalares do Sistema Único de Saúde (SIH-SUS), o Sistema de Informações de Agravos de Notificação (SINAN), a Relação Anual de Informações Sociais (RAIS), o Sistema de Informação de Comunicação de Acidentes de Trabalho (SISCAT), os boletins de ocorrência (BO), Guias de Encaminhamento Cadavérico (GEC) e Laudo de Exame Cadavérico (LEC). Para calcular a subnotificação de cada sistema, uma base de dados única foi criada com os registros de todas as fontes, eliminando-se repetições. Resultados: foram identificados 114 casos. Os subregistros encontrados foram: SIM, 28,9\%; SINAN, 44,7\%; SSP, 55,3\%; RAIS, 73,1\%; SISCAT, $65,4 \%$, para trabalhadores segurados. Conclusão: o melhor desempenho de registro é o do SIM. Observou-se extenso sub-registro em todas as demais fontes. Registros de mortalidade por ATF são subestimados. Ações de melhoria dos registros, especialmente no SIH-SUS, RAIS, SISCAT, SSP, seguidos pelo SINAN e SIM, são necessárias.

Palavras-chave: sub-registro; acidente de trabalho fatal; sistemas de informação.

Objective: to estimate the underreporting of fatal work accidents (FWA) in information systems of the Ministry of Health, State Secretariat of Public Security (SSP), Ministry of Labor and Social Security Secretariat, from 2007 to 2015, in Palmas, Tocantins. Methods: the data sources of the FWA cases were the Mortality Information System (SIM), the Hospital Information System of the Unified Health System (SIH-SUS), the Information System on Diseases of Compulsory Declaration (SINAN), the Annual Social Information Report (RAIS), the Information System of Communication of Work Accidents (SISCAT), police reports (PR), Cadaver Referrals (GEC) and Cadaveric Examination Reports (LEC). To calculate underreporting within each system, a single database was created with the records of all sources, eliminating repetitions. Results: 114 cases were identified. Under-reports identified were: SIM, 28.9\%; SINAN, 44.7\%; SSP, $55.3 \%$; RAIS, $73.1 \%$; SISCAT, $65.4 \%$, for insured workers. Conclusion: the best performance in registry is from SIM. Extensive underreporting was observed in all other sources. Mortality records due to FWA are underestimated. Action to improve recording, especially at SIH-SUS, RAIS, SISCAT, and SSP, followed by SINAN and SIM, are required.

Keywords: underreporting; fatal work accident; information systems. 


\section{Introdução}

A prevenção de acidentes de trabalho fatais (ATF) é um desafio em países em desenvolvimento, onde persistem altas taxa de mortalidade, apesar de serem reconhecidamente afetadas por extenso sub-registro. Oportunidades perdidas de informação refletem diretamente em menor capacidade na adoção de medidas de prevenção. Toda morte decorrente de um agravo à saúde evitável, como os ATF, representa falhas nas políticas e programas desenvolvidos para o seu controle. De acordo com dados da Organização Internacional do Trabalho (OIT), estima-se que em 2012 foram 320.580 mortes por acidentes de trabalho no mundo ${ }^{1}$. No Brasil, foram registrados 2.731 óbitos entre trabalhadores segurados em 2014, correspondendo a um coeficiente de mortalidade de 6,6/100.000 mil trabalhadores ${ }^{2}$, menor que o estimado para o estado de Tocantins, de $8,3 / 100.000 \mathrm{mil}^{3}$.

No Brasil, ATF são registrados em sistemas de informação em saúde e administrativos, sob a responsabilidade do Sistema Único de Saúde (SUS), Ministério do Trabalho e Secretaria da Previdência Social. Esses sistemas foram revisados recentemente por Galdino et al. ${ }^{4}$, que identificaram registros de ATF no âmbito das secretarias de segurança pública (SSP) de todas as unidades da federação, os quais, embora até o presente não se encontrem organizados em sistemas de informação digitais, com acesso público, constituem-se em fontes potenciais a serem exploradas. No SUS, registros de ATF provêm da Declaração de Óbito (DO), consolidados no Sistema de Informação sobre Mortalidade (SIM), nas Autorizações de Internação Hospitalar (AIH), no Sistema de Informações Hospitalares do SUS (SIH-SUS), e no Sistema de Informação de Agravos de Notificação (SINAN). Este último contém fichas de notificação e informações de agravos de interesse para a saúde do trabalhador, a exemplo dos acidentes de trabalho graves e acidentes de trabalho com exposição à materiais biológicos, de informação de violências (Sistema de Vigilância de Violências e Acidentes - Viva), intoxicações exógenas e acidentes com animais Peçonhentos, além das hepatites viRAIS e a de tétano. No Ministério do Trabalho, registros de ATF constam na Relação Anual de Informações Sociais (RAIS) e no Sistema de Informação de Comunicação de Acidentes de Trabalho (SISCAT), da Previdência Social ${ }^{4}$.

Devido à natureza violenta, mortes por causas externas requerem o registro e subsequente investigação de caráter jurídico-policial no âmbito da segurança pública. Isso ocorre inicialmente com o Boletim de Ocorrência (BO), seguido pela Guia de Encaminhamento Cadavérico (GEC) e Laudo de
Exame Cadavérico (LEC). BO e GEC são emitidos pelas delegacias de Polícia Civil e o LEC pelos institutos médicos legais (IML) ${ }^{5}$. O registro na secretaria de segurança pública deve ocorrer imediatamente após o conhecimento da ocorrência do óbito por causa não natural, seja no local do acidente, no hospital ou durante o transporte. Delegacias de polícia estão habilitadas para realizar esses registros, emitindo BO e GEC. Este último representa a autorização legal para o levantamento cadavérico (remoção do corpo), a realização de pericia e o transporte do corpo até o IML, onde é realizado exame cadavérico, com emissão da LEC, acompanhada da $\mathrm{DO}^{4}$.

Em cada um dos sistemas de informação mencionados, registros sobre ATF são reconhecidos como de pouca qualidade, incompletos e inconsistentes ${ }^{1}$. Concha-Barrientos et al. ${ }^{6}$ estimaram, para o ano de 2000, um sub-registro de ATF de 26,1\% em âmbito global. Nos Estados Unidos, Smith et al. ${ }^{7}$ identificaram ATF em múltiplas fontes de dados e encontraram sub-registro de 11\% em declarações de óbitos e $56 \%$ nos sistemas de benefícios de compensação de companhias de seguro. Em uma revisão de estudos brasileiros publicados entre 1994 e $2004^{8}$ verificou-se que declarações de óbito quando comparadas a entrevistas domiciliares apresentavam sub-registro de $81,9 \%{ }^{9}$ em Porto Alegre, de $27 \%$ em Campinas, e $83,4 \%$ no estado de São Paulo ${ }^{10}$. O sub-registro de BO em comparação ao SIM foi estimado em 71,1\% no Rio de Janeiro, em $1997^{11}$, valor próximo ao encontrado, de $78,4 \%$, quando BO foram comparados aos dados de um sindicato de trabalhadores da construção civil que abrangiam o período de 1997 e $2001^{12}$. Na zona norte da cidade de São Paulo, em 1998, do total de 32 casos de ATF registrados em BO, apenas 11 constavam na DO, estimando-se um sub-registro de $65,6 \%{ }^{13}$.

Registros de ATF nos sistemas de informação seguem fluxos de informação que estão sujeitos a barreiras e filtros, impedimentos parciais ou totais, que causam o sub-registro ${ }^{4}$. Azaroff et al. ${ }^{14}$ elencaram barreiras e filtros nos Estados Unidos, entre eles o receio em informar o acidente para a chefia por medo de retaliações como a redução do salário; falta de cobertura para assistência médico-hospitalar; evitar inspeções e fiscalizações; ou a pouca consciência sobre a importância dos registros e a incompletude nas informações hospitalares relacionadas ao atendimento dos trabalhadores acidentados. No estudo de Marsh e Jackson ${ }^{15}$, nos Estados Unidos, registros de ATF do Sistema de Mortalidade foram comparados aos do Censo de Acidentes de Trabalho Fatais, em 2003 e 2004, observando-se falhas nos códigos da Classificação Internacional de Doenças (CID-10) e nas narrativas sobre o óbito, especialmente no Sistema de Mortalidade. Sobre o campo na DO para registro da 
relação do óbito por causas externas com o trabalho, Iwamoto et al. ${ }^{16}$ encontraram 79,6\% dos dados registrados como ignorados em Uberaba, MG, entre 1997 e 2006. Não foram encontrados estudos com estimativas válidas de sub-registro de ATF no Tocantins.

Este estudo teve por objetivo estimar o sub-registro de acidentes de trabalho fatais (ATF) nos sistemas de informação do Ministério da Saúde, Secretaria Estadual de Segurança Pública (SSP), Ministério do Trabalho e Secretaria da Previdência Social, de 2007 a 2015, em Palmas, Tocantins. Espera-se que seus resultados contribuam para a melhoria dos registros, para a qualidade da informação e para ações de prevenção de ATF.

\section{Métodos}

Foi realizado um estudo de série dos casos de ATF registrados entre os anos de 2007 e 2015, em Palmas, Tocantins, com base em dados secundários. O período foi definido por representar a implantação do SINAN no registro de acidentes de trabalho, iniciada em 2007. A cidade de Palmas foi escolhida por conveniência para a coleta de dados.

Utilizaram-se os dados: do Ministério da Saúde, os sistemas de informação SIM, SIH-SUS e SINAN; do Ministério do Trabalho, a RAIS; da Secretaria da Previdência Social, o SISCAT; da SSP do Tocantins, os documentos BO, GEC e LEC, cujos acesso e uso foram autorizados para a pesquisa pela direção do IML. Dessas múltiplas fontes, construiu-se uma base de dados única contendo todos os casos com ao menos um registro em cada um dos sistemas e documentos listados e estimou-se o sub-registro de cada uma dessas fontes em relação à essa base única. A população de referência do estudo é a de trabalhadores ativos residentes na cidade de Palmas no período do estudo.

\section{Fontes de dados}

Sistema de Informação sobre Mortalidade (SIM) dados individuais anônimos estão disponíveis no portal Datasus, de onde foram extraídos: número do óbito, data de nascimento, data do óbito, sexo, ocupação registrada de acordo com os códigos da Classificação Brasileira de Ocupações (CBO), 2002, e a causa básica codificada pela Classificação Internacional das Doenças $10^{\mathrm{a}}$ revisão (CID-10). Esse sistema deveria cobrir todos os casos de óbito da população trabalhadora, independentemente do tipo de vínculo empregatício ou da situação no mercado de trabalho. O vínculo empregatício diz respeito à relação jurídica estabelecida entre duas partes (empregado e empregador), já a situação no mercado de trabalho refere-se ao tipo de vínculo e suas características de acordo com as fichas dos respectivos sistemas de informação. A extração se restringiu aos casos de acidente de trabalho com ocorrência no município de Palmas. O SIM tem cobertura universal e de acordo com a Organização Mundial da Saúde (OMS) tem qualidade regular ${ }^{17}$.

Sistema de Informações Hospitalares do SUS (SIH-SUS) - essa base de dados está disponível no portal do Centro Colaborador da Vigilância aos Agravos à Saúde do Trabalhador, www.ccvisat.ufba.br. Esse sistema deveria cobrir os casos de ATF de trabalhadores que foram hospitalizados. A extração foi limitada aos acidentes de trabalho ocorridos no período do estudo. Após a extração foram selecionados os registros referentes ao município de Palmas, que contavam com preenchimento adequado do campo de acidente de trabalho típico e de trajeto, com o número da $\mathrm{AIH}$, a data da internação, data do óbito e evolução da internação.

Sistema de Informação de Agravos de Notificação (SINAN) - integra vários subsistemas úteis para a identificação de ATF. Neste estudo foi utilizado apenas o sub-sistema de Acidentes de Trabalho Grave, disponível no portal do Centro Colaborador da Vigilância de Agravos à Saúde do Trabalhador (CCVISAT), de modo anônimo. Para os casos graves com óbito, esse sistema deveria cobrir a mesma população do SIM. Foram extraídos dados sobre data de nascimento, data do óbito, sexo, raça/cor, escolaridade, código do município de ocorrência, código de atividade econômica segundo a Classificação Nacional de Atividades Econômicas (CNAE), ocupação segundo o Código Brasileiro de Ocupações (CBO 2002), situação no mercado de trabalho, tempo de serviço na empresa, e evolução que define o desfecho óbito.

Boletim de Ocorrência (BO) - documento que registra a ocorrência de morte por causa violenta; todas as mortes desse tipo, por sua importância policial, são registradas em delegacias de polícia; no BO são relatadas as circunstâncias que permitem a identificação das causas do óbito e, quando pertinente, sua relação com o trabalho. Esses documentos encontram-se disponíveis apenas em versão impressa, armazenados em arquivos nas dependências do IML e possuem campos para a identificação da ocupação do trabalhador. Os documentos originais de cada uma das pastas individuais foram verificados para identificar se havia registro da causa básica de morte e da sua relação com o trabalho. Os dados dos casos identificados como ATF foram transcritos em uma planilha Excel, especificamente: nome, nome da mãe, data de nascimento, data do óbito, sexo, município de ocorrência e tipo de ocorrência (que registra se foi acidente de trabalho). 
Guia de Encaminhamento Cadavérico (GEC) após a emissão do BO, a delegacia de polícia precisa emitir autorização para a remoção do corpo, de responsabilidade dessa instituição, que o encaminha para o IML. Esses documentos possuem também a identificação da ocupação e a relação do acidente com o trabalho. Os arquivos foram consultados para a seleção dos ATF, dos quais foram registrados a numeração do GEC, nome, data de nascimento, data do óbito, sexo, município de ocorrência e características do acidente (que informa sobre a relação com o trabalho).

Laudo de Exame Cadavérico (LEC) - em seguida à necrópsia, o médico legista reúne todas as informações disponíveis no LEC, onde fica estabelecida a causa da morte, especificando a relação com o trabalho e a ocupação do trabalhador. Esses documentos foram consultados manualmente para identificação dos registros de ATF. Para cada caso foram digitados em planilha os seguintes dados: nome, data de nascimento, data do óbito e tipo de óbito (que identifica $o$ acidente de trabalho).

Relação Anual de Informações Sociais (RAIS) esse sistema registra informações sociais e econômicas fornecidas anualmente ao Ministério do Trabalho pelas empresas, públicas e privadas, que contratam trabalhadores em regime pela Consolidação das Leis Trabalhistas (CLT). Agrega informações sobre a atividade de trabalho e características de cada empregado, inclusive se houve terminação do contrato e por qual motivo, especificando se foi devido à morte por acidente de trabalho. Esse sistema está disponível para acesso exclusivo a pesquisadores, mediante solicitação e aprovação por comitê de ética em pesquisa. A cobertura desse sistema inclui apenas o registro de trabalhadores com vínculos empregatícios e contratos celetistas. Analisaram-se os dados sobre: nome, data de nascimento, data de óbito, sexo, município de ocorrência e terminação do contrato por motivo de óbito por acidente de trabalho.

Sistema de Informação de Comunicação de Acidentes de Trabalho (SISCAT) - esse sistema registra dados de todos os trabalhadores segurados pela Previdência e cobertos pelo Seguro Acidente de Trabalho (Riscos de Acidente de Trabalho, RAT) e as Comunicações de Acidentes de Trabalho (CAT) junto ao Instituto Nacional do Seguro Social (INSS), que possibilitam o afastamento do trabalhador acidentado. As informações extraídas desse sistema para compor a base única de dados foram: nome, data de nascimento, data de óbito, sexo, município de ocorrência do acidente. Nesse sistema, a cobertura inclui apenas os segurados e trabalhadores celetistas.
Essas duas bases de dados, RAIS e SISCAT, encontravam-se disponíveis para estudos no Instituto de Saúde Coletiva da Universidade Federal da Bahia (ISC-UFBA).

Para o pareamento dos registros, correspondentes a um único indivíduo, foram identificadas as seguintes variáveis: data de nascimento, data do óbito e sexo. Outras variáveis descritoras também foram definidas: raça/cor (branca, parda, preta, indígena, amarela); faixa de idade em anos (18 a 29, 30 a 49, maior que 50); escolaridade em anos (nenhum, 1 a 7 , 8 a 11, 12 ou mais); ocupação codificada pela CBO 2002; atividade econômica codificada pela CNAE; situação no mercado de trabalho (formal ou informal); e causa do óbito codificada pelo CID 10. Para cada um dos casos encontrados nos sistemas/documentos, foi criada uma variável correspondente à base onde se encontrava registrada.

\section{Análise}

A partir da base única construída por meio da similaridade das variáveis identificadoras de cada registro, após a eliminação de repetidos, estimaram-se as frequências absolutas e relativas das variáveis descritoras. Calcularam-se as proporções de co-ocorrência de registros em diferentes sistemas de informação, para as quais a proporção complementar indica a magnitude do sub-registro daquele sistema. A análise foi realizada empregando-se planilhas Excel e SAS 9.4. A apresentação dos resultados não permite a identificação dos casos individualmente. O projeto foi registrado na Plataforma Brasil e aprovado pelo Comitê de Ética em Pesquisa do Instituto de Saúde Coletiva da Universidade Federal da Bahia (Parecer n ${ }^{\circ}$ 2.088.289, 16/05/2017).

\section{Resultados}

Após o pareamento dos casos de todos os sistemas de informação, ficaram 114 óbitos identificados como ATF, dos quais quase a totalidade foi do sexo masculino $(93,8 \%)$, a maioria da raça/cor parda $(54,4 \%)$, com grande parte entre 30 a 49 anos idade $(46,5 \%)$. As causas de morte mais comuns foram os acidentes de transporte envolvendo veículos automotores $(54,4 \%)$, quedas (20\%) e eletrocussão (16\%), em trabalhadores dos ramos da construção (13\%) e agricultura (13\%), com a ocupação de pedreiros e serventes (17\%), eletricistas (10,) e agricultores (13\%) (Tabela 1). Apenas sete casos eram de mulheres, todos envolvendo veículo a motor, notando-se que para três houve registro apenas no SINAN. 
Tabela 1 Características sociodemográficas e ocupacionais dos acidentes de trabalho fatais identificados nos sistemas de registro do Ministério da Saúde, Ministério do Trabalho e Secretaria Estadual de Segurança Pública, ocorridos em Palmas, Tocantins, 2007-2015

\begin{tabular}{|c|c|c|}
\hline Características & $N=114$ & $\%$ \\
\hline \multicolumn{3}{|l|}{$\operatorname{Sexo}(n=114)$} \\
\hline Masculino & 107 & 93,8 \\
\hline Feminino & 7 & 6,2 \\
\hline \multicolumn{3}{|l|}{ Raça/cor $(n=114)$} \\
\hline Branca & 24 & 21,0 \\
\hline Preta & 12 & 10,5 \\
\hline Parda & 62 & 54,4 \\
\hline Outras & 16 & 14,1 \\
\hline \multicolumn{3}{|l|}{ Faixa de idade em anos $(n=114)$} \\
\hline $18-29$ & 38 & 33,4 \\
\hline $30-49$ & 53 & 46,5 \\
\hline$\geq 50$ & 23 & 20,1 \\
\hline \multicolumn{3}{|l|}{ Escolaridade em anos $(n=48)$} \\
\hline Nenhum & 4 & 8,4 \\
\hline $1-7$ & 21 & 43,7 \\
\hline $8-11$ & 14 & 29,2 \\
\hline$\geq 12$ & 9 & 18,7 \\
\hline \multicolumn{3}{|l|}{ Ocupação (n = 99) } \\
\hline Pedreiro/servente de obras & 17 & 17,2 \\
\hline Produtor agrícola/trabalhador agropecuário/ tratorista agrícola & 13 & 13,1 \\
\hline Motofretista/motorista de caminhão/motorista de carro de passeio/motorista de táxi & 12 & 12,1 \\
\hline Eletricista de instalações/eletricista de manutenção de redes elétricas & 10 & 10,1 \\
\hline Outras & 47 & 47,5 \\
\hline \multicolumn{3}{|l|}{ Ramo de atividade econômica $(\mathrm{n}=49)$} \\
\hline Construção & 13 & 26,5 \\
\hline Agropecuária & 13 & 26,5 \\
\hline Indústria da transformação & 7 & 14,3 \\
\hline $\begin{array}{l}\text { Comércio e serviços de reparação de veículos automotores, objetos pessoais e } \\
\text { domésticos }\end{array}$ & 5 & 10,2 \\
\hline Outras & 11 & 22,5 \\
\hline \multicolumn{3}{|l|}{ Causa do óbito (Grupo CID 10) ( $n=114)$} \\
\hline Acidente envolvendo veículos & 62 & 54,4 \\
\hline Quedas & 20 & 17,6 \\
\hline Exposição a corrente elétrica & 16 & 14,0 \\
\hline Outras causas & 16 & 14,0 \\
\hline
\end{tabular}

Foram encontrados 81 casos no SIM, 63 no SINAN, nenhum caso no SIH-SUS, 51 registros em documentos da SSP, sete na RAIS e, nove registros no SISCAT (Tabela 2). Comparando-se dados de cada sistema de informação com a base única de 114 casos, estimou-se que o sub-registro do ATF no SIM foi de 28,9\%, no SINAN $44,7 \%$, e de $55,3 \%$ no SSP. Na RAIS o sub-registro foi de 73,1\%, enquanto no SISCAT foi de $65,4 \%$ (Tabela 2). A distribuição dos registros isolados e simultâneos nas diversas bases de dados é mostrada na Tabela 3. Observa-se que apenas 26 mortes foram identificadas exclusivamente no SIM (22,8\%), 24 somente no SINAN (21,1\%), enquanto o registro simultâneo nessas duas bases universais ocorreu apenas para 13 casos $(11,4 \%)$; considerando que o esperado é o registro comum, estimou-se em $88,6 \%$ a sua falta. Não foram encontrados casos de ATF no SIH-SUS. Nos documentos da SSP (Tabela 3), apenas quatro $(3,5 \%)$ casos foram identificados exclusivamente, com três relatos em LEC $(2,6 \%)$, e somente um registro simultâneo em BO e LEC (0,9\%). Nenhum ATF foi registrado em todas as bases, i.e., SIM, SIH-SUS, SINAN e documentos da SSP ou na RAIS e SISCAT. 
Tabela 2 Distribuição dos casos de acidentes de trabalho fatais identificados nos sistemas de registro do Ministério da Saúde, Ministério do Trabalho e Secretaria Estadual de Segurança Pública, por sistema de informação de origem, e estimativas de sub-registro. Palmas, Tocantins, 2007-2015

\begin{tabular}{lcc}
\hline Sistemas de informação & N=114 & Sub-registro (\%) \\
\hline Sistema de Informação sobre Mortalidade - SIM & 81 & 28,9 \\
Sistema de Informações Hospitalares - SIH-SUS & 0 & -- \\
Sistema de Informação de Agravos de Notificação - SINAN & 63 & 44,7 \\
Secretaria de Segurança Pública - SSP & 51 & 55,3 \\
Relação Anual de Informações Sociais - RAIS & 7 & 73,1 \\
Sistema de Informação de Comunicações de Acidente de Trabalho - SISCAT & 9 & 65,4 \\
\hline
\end{tabular}

Tabela 3 Distribuição dos registros de acidentes de trabalho fatais identificados em sistemas de informação universais do Ministério da Saúde e da Secretaria Estadual de Segurança Pública (SSP). Palmas, Tocantins, 2007-2015

\begin{tabular}{|c|c|c|}
\hline Especificação* & $N=114$ & $\%$ \\
\hline \multicolumn{3}{|l|}{ Sistemas de informação em Saúde } \\
\hline Somente Sistema de Informação sobre Mortalidade, SIM & 26 & 22,8 \\
\hline Somente Sistema de Informação de Agravos de Notificação, SINAN & 24 & 21,1 \\
\hline Somente SIH-SUS & 0 & --- \\
\hline Ambos SIM e SINAN & 13 & 11,4 \\
\hline Subtotal & 63 & 55,3 \\
\hline \multicolumn{3}{|l|}{ Registros da SSP } \\
\hline Boletim de Ocorrência, BO & 0 & -- \\
\hline Guia de Encaminhamento Cadavérico, GEC & 0 & --- \\
\hline Laudo de Exame Cadavérico, LEC & 3 & 2,6 \\
\hline Ambos BO e GEC & 0 & -- \\
\hline Ambos BO e LEC & 1 & 0,9 \\
\hline BO, GEC e LEC & 0 & -- \\
\hline GEC e LEC & 0 & -- \\
\hline Subtotal & 4 & 3,5 \\
\hline \multicolumn{3}{|l|}{ Sistemas de informação em Saúde e da SSP } \\
\hline SIM, SINAN, BO, GEC, LEC & 6 & 5,3 \\
\hline SIM, BO & 4 & 3,5 \\
\hline SIM, LEC & 0 & -- \\
\hline SIM, GEC & 0 & -- \\
\hline SIM, BO, LEC & 12 & 10,5 \\
\hline SIM, BO, GEC & 1 & 0,9 \\
\hline SIM, BO, GEC, LEC & 1 & 0,9 \\
\hline SIM, LEC & 3 & 2,6 \\
\hline SIM, LEC, GEC & 0 & -- \\
\hline SIM, SINAN, BO & 6 & 5,3 \\
\hline SIM, SINAN, BO, LEC & 9 & 7,9 \\
\hline SINAN, BO & 0 & -- \\
\hline SINAN, BO, GEC & 0 & -- \\
\hline SINAN, BO, LEC & 3 & 2,6 \\
\hline SINAN, BO, GEC, LEC & 2 & 1,7 \\
\hline Subtotal & 47 & 41,2 \\
\hline
\end{tabular}

*Dados da RAIS e SISCAT foram omitidos por serem pequenos e para trazerem clareza para a apresentação. 
Na Figura 1, apresentam-se para os casos encontrados no SIM ( $\mathrm{n}=81$ ) os registros comuns em BO (46,9\%), e destes, oito apareciam na GEC (21,1\%), e sete nos LEC $(87,5 \%)$. Em relação ao SIM, o sub-registro do $\mathrm{BO}$ é de 53,1\% (a soma dos não registrados e ausentes), 90,1\% do GEC e 91,3\% do LEC. Entre os óbitos de trabalhadores registrados que compunham a base única $(n=26)$, apenas sete foram registrados na RAIS e nove no SISCAT, estimando-se sub-registro de 73,1\% e 65,4\%, respectivamente (Figura 2).

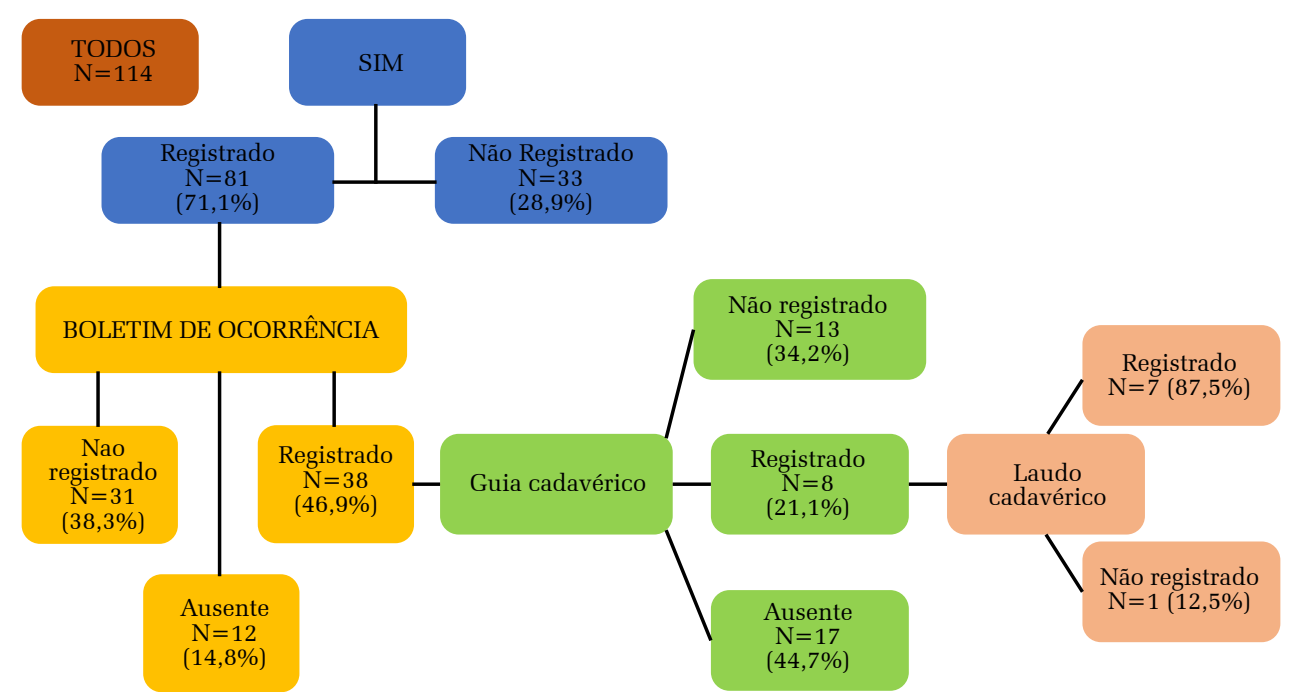

Figura 1 Registros de acidentes de trabalho fatais em sistemas de informação e documentos da Secretaria Estadual de Segurança Pública entre os casos identificados no SIM. Palmas, Tocantins, 2007-2015

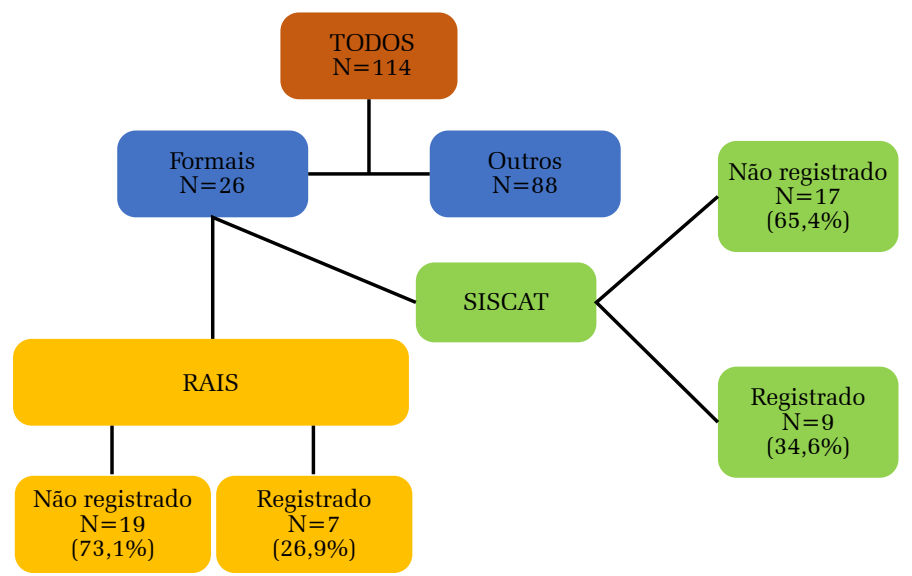

Figura 2 Distribuição dos registros de acidentes de trabalho fatais na RAIS e no SISCAT, para trabalhadores formais, em Palmas, Tocantins, 2007-2015

\section{Discussão}

Os sistemas analisados neste estudo possuem diferentes coberturas. SIM, SINAN, BO, GEC e LEC deveriam incluir todos os casos de óbitos de trabalhadores, independentemente do vínculo empregatício ou da situação no mercado de trabalho. O SIH-SUS deveria conter todos os casos em que ocorreu hospitalização, entre os registrados nos sistemas citados anteriormente. Para RAIS e SISCAT, sistemas ligados ao Ministério do Trabalho, a cobertura limita-se aos segurados da previdência e aos trabalhadores celetistas, o que poderia explicar parte do sub-registro dos ATF.

Os achados deste estudo mostram que existe elevado sub-registro de ATF em Palmas, Tocantins, nos sistemas SIM, SIH-SUS (não houve registro), SINAN e nos documentos da SSP, na RAIS e no SISCAT. As 
maiores estimativas de sub-registro, considerando os 114 casos da base única, ocorreram na RAIS $(73,1 \%)$ e SISCAT $(65,4 \%)$, documentos da SSP $(53,3 \%)$, seguida pelo SINAN $(44,7 \%)$, enquanto o SIM teve a menor $(28,9 \%)$. Foi bem elevado o sub-registro em ambos SIM e SINAN, estimado em 89\%. Não foi identificado nenhum caso que tivesse sido registrado em todas as bases investigadas.

O sub-registro de ATF estimado neste estudo para o SIM $(28,9 \%)$ não difere expressivamente do valor $(26,1 \%)$ relatado para estatísticas mundiais em $2000^{6}$ e que pouco variou entre as regiões, de $27 \%$ a $28 \%{ }^{6}$. Tais estimativas foram maiores do que as de sub-registro de $10 \%$ a $33 \%$ apresentadas para os Estados Unidos, 28\% na África do Sul e 23\% na Austrália. Nos Estados Unidos, ATF causados por eletrocussões, quedas e máquinas foram sub-registrados em $11 \%$ nos atestados de óbitos, $41 \%$ em registros das autoridades de segurança do trabalho e $56 \%$ em sistemas de compensação ${ }^{7}$. Na Austrália, entre 1989-1992, calculou-se em 34\% o sub-registro de ATF em sistemas de compensação e de instituições de saúde e segurança no trabalho ${ }^{18}$. $\mathrm{O}$ achado deste estudo, de $28,9 \%$ de sub-registro no SIM, também ficou próximo das estimativas de $27,8 \%$ em Belo Horizonte, no ano de $2011^{19}$, porém, menor que $48,9 \%$ estimado por outro estudo para o mesmo município, entre 2008 e $2010^{20}$.

Portanto, o sub-registro de ATF no SIM encontrado neste estudo para o município de Palmas, revelou-se próximo aos estudos supracitados. Isto revela ser resultante dos esforços do Ministério da Saúde em melhorar a cobertura e qualidade desse sistema, que tem apresentado outras evidências de avanços, como na redução de causas mal definidas de óbitos ${ }^{23}$. Ressalta-se também esforços do Ministério da Saúde em relação à ampliação e o fortalecimento da Rede Nacional de Atenção Integral à Saúde do Trabalhador (Renast). Especificamente no Tocantins, de acordo com informações dos relatórios anuais de gestão, a Secretaria Estadual de Saúde vem oferecendo treinamentos voltados para os profissionais responsáveis pelo preenchimento da DO, em especial, para os casos definidos como causas externas, valorizando a vigilância do óbito. Destaca-se que a ampliação da Renast no Tocantins se deu com a implantação dos Núcleos de Saúde do Trabalhador em mais de 20 municípios, responsáveis pela execução de diversas ações de prevenção relacionadas à saúde do trabalhador.

Neste estudo, o SINAN apresentou sub-registro de ATF de 44,7\% em relação à base única, próximo ao achado de $54,6 \%{ }^{20}$ de um estudo conduzido em Belo Horizonte, mas muito maior que $14,8 \%{ }^{19}$ estimado também em Belo Horizonte, entre 2008 e 2010. Essa comparação deve ser vista com cautela porque o subsistema Acidentes de Trabalho Graves do SINAN teve sua implantação iniciada em 2007, apresentando ao longo dos anos seguintes crescimento da cobertura, diferenciado de acordo com a unidade da federação e região ${ }^{24}$. O sub-registro no SINAN pode indicar também a pouca importância que ainda lhe é atribuída entre os profissionais do SUS e ao limite das notificações de ATF, que eram registrados apenas em Unidades Sentinelas, com base na Portaria do Ministério de Saúde 1.271 de 6 de junho de 2014, norma modificada para cobertura universal apenas em 2014.

Os documentos da SSP mostraram elevado sub-registro neste estudo, maior que a estimativa de $24,1 \%$ (13 em 54 casos) encontrada em Belo Horizonte ${ }^{19}$, embora, neste estudo, baseada apenas nos LEC. No estudo de Carneiro ${ }^{13}$ realizado em São Paulo, no ano de 1998, o sub-registro de ATF da DO comparada aos dados dos BO foi estimado em 65,6\%. Todavia, com dados do Rio de Janeiro, de 1997, o sub-registro do $\mathrm{BO}$ em relação à DO foi de $71,1 \%^{11}$. Não foram encontrados estudos sobre o sub-registro do BO em relação ao SINAN. Calculou-se sub-registro de ATF em 90,1\% nas GEC em relação as DO, indicando que a investigação policial não está buscando identificar a relação do óbito com o trabalho. Ainda que no BO, GEC e LEC existam os campos "ocupação" e "tipo e local do acidente", na maioria dos casos não há preenchimento. A coleta de provas por agentes policiais no local do acidente por ocasião da remoção do corpo é essencial na investigação da causa do óbito. No Brasil, apenas uma pequena parte de mortes violentas é investigada e tem esclarecida a sua autoria e motivação ${ }^{13}$. Esse grande sub-registro de ATF nos documentos da SSP pode ter as mesmas causas relacionadas ao trabalho, além de possível falta de conhecimento das equipes das delegacias de polícia sobre a importância dessa informação para a Saúde Pública e para os familiares da vítima. Outra possível explicação para esse elevado sub-registro na SSP de ATF é que, casos suspeitos de acidentes de trabalho podem não contar com provas ou evidências suficientes, ou falte o conhecimento de parte dos agentes sobre a definição formal de acidente de trabalho. É comum os registros de SSP não considerarem como ATF casos de óbitos de motoristas profissionais durante sua jornada de trabalho, ou agressões interpessoais, como estupro, ou aborto provocado por situações de trabalho ${ }^{13}$. No geral, as causas de sub-registro parecem também residir na dificuldade de obtenção de provas, que podem ser ocultadas pela empresa, e na cultura disseminada de que a culpa é da vítima. Nesse sentido, é importante verificar a melhoria que foi instituída nos registros da SSP no Tocantins com a inserção, em 2010, do campo acidente de trabalho na GEC. 
A partir das informações constantes no SINAN, foi possível identificar se o tipo de vínculo empregatício do acidentado era formal (registrado em carteira de trabalho) e, assim, rastrear os casos no SISCAT, sistema de responsabilidade da Previdência Social. $\mathrm{O}$ achado mais surpreendente foi o grande sub-registro de ATF no SISCAT, cujos dados de acidentes são assumidos como os de melhor qualidade e geralmente são os apresentados como estatísticas oficiais do Brasil nas publicações da OIT. Resultados parecidos com os citados anteriormente foram encontrados em um estudo ${ }^{22}$ em Minas GeRAIS, conduzido em 1999, com sub-registro de 66,6\%. Quase duas décadas depois, é possível que essa realidade tenha se modificado, mas não em Palmas, Tocantins. É possível que trabalhadores dessa região, ainda considerada afastada e remota, não tenham conhecimento sobre seus direitos ou não se reconheçam com capacidade de reivindicá-los, buscando o registro dos ATF. É cruel a realidade vivida por trabalhadores jovens, que morrem por causas ocupacionais e encontram-se desprovidos dos seus direitos básicos, como o de ter um ambiente de trabalho seguro e saudável. É ainda mais cruel, quando mesmo sendo contribuintes da Previdência, com contratos de trabalho formal e cobertos pelo Seguro Acidente de Trabalho, ao morrerem por acidente de trabalho suas famílias necessitadas podem não estar recebendo as devidas pensões e indenizações, como indica o elevado sub-registro no SISCAT. Não emitir e nem comunicar o acidente, via CAT, constitui infração à legislação previdenciária, sujeita a aplicação de penalidade (multa), muito embora não se possa afastar problemas na completitude de registros apesar do possível recebimento de benefícios compensatórios sem registro junto a previdência.

Este estudo é provavelmente o primeiro a analisar diversas fontes de registros de ATF em sistemas de informação do Brasil, no caso, para a área do município de Palmas, Tocantins. Este estudo apresenta limitações referentes ao pequeno número de casos para análise. Outras limitações são a impossibilidade de analisar a evolução, ao longo do tempo, do desempenho dos sistemas de informação para registros de ATF. Também, deve-se considerar que a base única utilizada como referência pode estar incompleta, por perdas de registros nos sistemas e documentos analisados, muito embora a situação de Palmas, Tocantins, especialmente o baixo sub-registro do SIM possa ser distinta das demais regiões. Como evidência disso, estudos $^{9,21}$ realizados no Brasil mostraram estimativas bem mais elevadas de sub-registro quando compararam dados do SIM com relatos obtidos em entrevistas domiciliares.

As diferenças de sub-registro de ATF entre os sistemas de informação assinalam para a necessidade de melhoria da qualidade e cobertura da informação visando a melhoria da prevenção. É necessário que casos de ATF sejam identificados e melhor registrados pelos órgãos do âmbito jurídico-policial e que dados dos sistemas de informação da SSP se tornem públicos e acessíveis, com o devido anonimato para garantir os princípios éticos no tratamento da privacidade dos sujeitos. A criação de um fluxo de informação de dados de ATF entre os setores de vigilância em saúde em todos os níveis do SUS, Ministério do Trabalho, Previdência Social e SSP pode contribuir na redução do sub-registro em todas as bases. Todavia, a vigilância e a adoção imediata de ações visando à proteção do trabalhador requer a pronta comunicação entre Secretaria da Saúde e delegacias de polícia. A inclusão do campo "acidente de trabalho" nos BO e LEC, inserindo algumas perguntas sobre a relação com o trabalho, também pode contribuir na identificação de óbitos por AT. O sub-registro do SINAN, de quase metade da base comum deste estudo, é elevado e inaceitável, e requer medidas urgentes para sua superação, pois essa base é importante para o acesso a mais e melhores informações sobre todos os casos de ATF. Espera-se que registros simultâneos em sistemas de informação em saúde ocorram para todos os casos de óbitos de ATF.

Este estudo mostrou que os casos de óbitos por acidente de trabalho não são simultaneamente registrados por todos os sistemas, sendo necessária e urgente a integração entre eles. O sub-registro de acidentes de trabalho de qualquer sistema de informação contribui para a invisibilidade da real extensão desse trágico evento, evitável na maioria das situações. Mais do que um problema metodológico, de precisão de dados, este é um problema social e político, ao refletir possível negligência de parte dos gestores e comprometer todos os níveis de cuidado à saúde, da segurança pública e da proteção social via previdência. Esse problema pode também revelar o não cumprimento de direitos constitucionais de grande parte da população de trabalhadores do país.

\section{Contribuições de autoria}

As autoras elaboraram conjuntamente o projeto, a análise dos dados e redação do manuscrito. A coleta de dados foi realizada por Rodrigues AB. 


\section{Referências}

1. Takala J, Hämäläinen P, Saarela KL, Yun LY, Manickam K, Jin TW, et al. Global estimates of the burden of injury and illness at work in 2012. J Occup Environ Hyg. 2014;11(5):326-37.

2. Brasil. Ministério da Fazenda. Anuário Estatístico da Previdência Social: AEPS, 2015 [Internet]. [acesso em 25 mar 2019]. Disponível em: http://sa.previdencia. gov.br/site/2015/08/AEPS-2015-FINAL.pdf

3. Brasil. Ministério da Fazenda. Anuário Estatístico de Acidente de Trabalho: AEAT, 2015 [Internet]. [acesso em 25 mar 2019]. Disponível em: http://sa.previdencia.gov.br/site/2017/05/ aeat15.pdf

4. Batista AG, Santana VS, Ferrite S. Registro de dados sobre acidentes de trabalho fatais em sistemas de informação no Brasil. Ciênc Saúde Coletiva. 2019;24(3):693-704.

5. Brasil. Ministério da Justiça. Lei no 12.681 de 4 de julho de 2012. Institui o Sistema Nacional de Informações de Segurança Pública, Prisionais e sobre Drogas - SINESP. Diário Oficial da União. 4 jul 2012. [acesso em 15 jul 2017]. Disponível em: http://www.planalto.gov.br/ccivil_03/_Ato20112014/2012/Lei/L12681.htm.

6. Concha-Barrientos M, Nelson DI, Fingerhut M, Driscoll T, Leigh J. The global burden due to occupational injury. Am J Ind Med. 2005;48(6):470-81.

7. Smith GS, Veazie MA, Benjamin KL. The use of sentinel injury deaths to evaluate the quality of multiple source reporting for occupational injuries. Ann Epidemiol. 2005;15(3):219-27.

8. Santana V, Nobre L, Waldvogel BC. Acidentes de trabalho no Brasil entre 1994 e 2004: uma revisão. Ciênc Saúde Coletiva. 2005;10(4):841-55.

9. Oliveira PAB, Mendes JM. Acidentes de trabalho: violência urbana e morte em Porto Alegre, Rio Grande do Sul, Brasil. Cad Saúde Púb. 1997;13:73-83.

10. Hennington EA, Cordeiro R, Moreira Filho DC. Trabalho, violência e morte em Campinas, São Paulo, Brasil. Cad Saúde Púb. 2004;20(2):610-17.

11. Pepe CCCA. Estratégias para superar a desinformação: um estudo sobre os acidentes de trabalho fatais no Rio de Janeiro [dissertação]. São Paulo (SP): Fundação Oswaldo Cruz; 2002.

12. Mangas RMN, Gomez CM, Thedim-Costa SMF. Acidentes de trabalho fatais e desproteção social na indústria da construção civil do Rio de Janeiro. Rev Bras Saúde Ocup. 2008;33(118):48-55.
13. Carneiro SAM. Trabalho e violência: relação de proximidade: violência a trabalhadores durante jornada de trabalho, na Zona Norte de São Paulo, em 1998 [dissertação]. São Paulo: Universidade de São Paulo; 2000.

14. Azaroff LS, Levenstein C, Wegman DH. Occupational injury and illness surveillance: conceptual filters explain underreporting. Am J Public Health. 2002; 92(9):1421-9.

15. Marsh SM, Jackson LL. A comparison of fatal occupational injury event characteristics from the Census of Fatal Occupational Injuries and the Vital Statistics Mortality System. J Safety Res. 2013;46:119-25.

16. Iwamoto HH, Camargo FC, Tavares LC, Miranzi SSC. Acidentes de trabalho fatais e a qualidade das informações de seus registros em Uberaba, em Minas GeRAIS e no Brasil, 1997 a 2006. Rev Bras Saúde Ocup. 2011;36(124):208-15.

17. Pan American Health Organization. Health situation in the Americas: 20 years basic indicators. 2014. Washington, D.C.: WHO; 2014.

18. Driscoll T, Mitchell R, Mandryk J, Healey S, Hendrie L, Hull B. Coverage of work related fatalities in Australia by compensation and occupational health and safety agencies. Occup Environ Med. 2003;60(3):195-200.

19. Bordoni PHC, Bordoni LC, Silva JM, Drumond EF. Utilização do método de captura-recaptura de casos para a melhoria do registro dos acidentes de trabalho fatais em Belo Horizonte, Minas GeRAIS, 2011. Epidemiol Serv Saúde. 2016;25(1):85-94.

20. Drumond EF, Silva JM. Avaliação de estratégia para identificação e mensuração dos acidentes de trabalho fatais. Ciên Saúde Coletiva. 2013;18(5):1361-5.

21. Binder MCP, Cordeiro R. Sub-registro de acidentes do trabalho em localidade do Estado de São Paulo, 1997. Rev Saúde Pública. 2003;37(4):409-16.

22. Correa PRL, Assunção AA. A subnotificação de mortes por acidentes de trabalho: estudo de três bancos de dados. Epidemiol Serv Saude. 2003;12(4):203-12.

23. Ferreira-De-Sousa FN, Santana VS. Mortalidade por acidentes de trabalho entre trabalhadores da agropecuária no Brasil, 2000-2010. Cad Saúde Pública. 2016;32(4): e00071914.

24. Galdino A, Santana VS, Ferrite S. Os Centros de Referência em Saúde do Trabalhador e a notificação de acidentes de trabalho no Brasil. Cad de Saúde Pública. 2012;28(1):145-59. 